\title{
Diagnostic Yield and Outcomes of Computed Tomography of the Head in Critically III Nontrauma Patients
}

Journal of Intensive Care Medicine 2019, Vol. 34(II-I2) 955-966

(C) The Author(s) 2017

Article reuse guidelines:

sagepub.com/journals-permissions DOI: $|0.1| 77 / 08850666|772090|$

journals.sagepub.com/home/jic

(S)AGE

\author{
Fabian Finkelmeier, MD', Sophie Walter', Kai-Henrik Peiffer, MD', Anjali Cremer, MD², \\ Andrea Tal, MD', Thomas Vogl, MD, PhD ${ }^{3}$, Stefan Zeuzem, MD, PhD', \\ Stephan Fichtlscherer, MD, PhD $^{4}$, Mireen Friedrich-Rust, MD, PhD', \\ Jörg Bojunga, MD, PhD', and Harald Farnik, MD, PhD'
}

\begin{abstract}
Background: Computed tomography of the head (HCT) is a widely used diagnostic tool, especially for emergency and trauma patients. However, the diagnostic yield and outcomes of HCT for patients on medical intensive care units (MICUs) are largely unknown. Methods: We retrospectively evaluated all head CTs from patients admitted to a single-center MICU during a 5-year period for CT indications, diagnostic yield, and therapeutic consequences. Uni- and multivariate analyses for the evaluation of risk factors for positive head CT were conducted. Results: Six hundred ninety (18.8\%) of all patients during a 5-year period underwent HCT; $78.7 \%$ had negative CT results, while $21.3 \%$ of all patients had at least I new pathological finding. The main indication for acquiring CT scan of the head was an altered mental state (AMS) in $23.5 \%$, followed by a new focal neurology in $20.7 \%$ and an inadequate wake up after stopping sedation in $14.9 \%$ of all patients. The most common new finding was intracerebral bleeding in $6.4 \%$. In $6.7 \%$, the CT scan itself led to a change of therapy of any kind. Admission after resuscitation or a new focal neurology were independent predictors of a positive CT. Psychic alteration and AMS were both independent predictors of a higher chance of a negative head CT. Positive HCT during MICU is an independent predictor of lower survival. Conclusions: New onset of focal neurologic deficit seems to be a good predictor for a positive CT, while AMS and psychic alterations seem to be very poor predictors. A positive head CT is an independent predictor of death for MICU patients.
\end{abstract}

\section{Keywords}

computed tomography, CT, critical ill patients, critical care unit, sepsis

\section{Background}

The use of computed tomography (CT) is rapidly increasing in clinical and nonclinical settings since its development in 1972. In 1980, an estimate of 3 million CT scans in the United States per year were obtained, while 25 years later, the number grew to above 60 million CT scans. Most of the CT scans are done in adults, and around one-third of all adult scans are scans of the head. ${ }^{1}$ There is an increasing awareness of risks associated with radiation exposure; however, dose reduction techniques reduced effective doses to less than the annual natural background radiation, making the decision to perform a CT scan even more easy. ${ }^{2}$

Intensive care unit (ICU) patients are sometimes hard to evaluate with clinical tools as they are often intubated or show neurological deterioration from unknown causes. Multiple reasons could lead to warrant head CT scan (HCT) such as onset of a new focal neurology, failure to wake up after sedation, or probably most common an altered mental state (AMS). Head CT scans yield inherent risks such as radiation ${ }^{1,3,4}$ or especially for ICU patients intrahospital transport ${ }^{5,6}$; furthermore, reducing costs for the health-care system is a growing issue. ${ }^{7}$

Increasing data exist on patients in emergency units, ${ }^{8}$ for example, patients with syncope ${ }^{9-12}$ or patients with minor head

\footnotetext{
'Medizinische Klinik I, Gastroenterologie, Universitätsklinikum Frankfurt, Goethe-Universität, Frankfurt, Germany

${ }^{2}$ Medizinische Klinik 3, Hämato-Onkologie, Universitätsklinikum Frankfurt, Goethe-Universität, Frankfurt, Germany

${ }^{3}$ Diagnostische und Interventionelle Radiologie, Universitätsklinikum Frankfurt, Goethe-Universität, Frankfurt, Germany

${ }^{4}$ Medizinische Klinik 2, Kardiologie, Universitätsklinikum Frankfurt, Goethe-Universität, Frankfurt, Germany
}

Received April 21, 2017. Received revised June 17, 2017. Accepted for publication June 26, 2017.

\section{Corresponding Author:}

Fabian Finkelmeier, Department of Internal Medicine I, Frankfurt University Hospital, Theodor-Stern-Kai 7, D-60590 Frankfurt, Germany.

Email: fabian.finkelmeier@kgu.de 
trauma. ${ }^{13-15}$ Most of these studies show a low diagnostic yield, and therefore, clinical and prognostic factors to choose the right patients for CT scans are warranted. ${ }^{16,17}$ Especially HCTs in unselected medical patients seem to be of low diagnostic yield as shown by Owlia et al, who found only $4 \%$ of all HCTs yielding significant findings. ${ }^{17}$

For surgical patients, especially neurosurgical patients, it may be easier to define indications for CT scans, however, this does not account for medical ICU (MICU) patients often presenting with multifactorial clinical conditions like headache, syncope, AMS, psychic alterations, and other risk factors such as coagulopathy and age. Interestingly, data from patients on MICUs are scarce, and we could only identify 5 studies so far dealing with this subgroup of patients. ${ }^{18-22}$ However, these studies mostly included a mix of surgical and medical patients or analyzed special subcohorts of patients.

The aim of this study was to evaluate why and for which patients a CT scan of the head is requested in a large cohort of patients on an MICU and the diagnostic and therapeutic yields of this procedure. Furthermore, we aimed at evaluating risk factors for a positive CT and whether positive HCT is a relevant survival factors on MICU.

\section{Methods}

\section{Selection of Patients}

By a systematic query of the University Hospital Frankfurt clinical database, all patients admitted to the MICU of the Frankfurt University Hospital, a tertiary clinic in a large metropolitan area, between January 2010 and August 2015 were included into this retrospective cohort study. The study was performed in accordance with the 1975 Declaration of Helsinki. The study was approved by the ethical committee of the Frankfurt University Hospital (protocol number 535/15).

The MICU is a specialized ward with 16 intensive care beds for exclusively patients of the internal medicine departments and patients with nontraumatic diseases requiring intensive care. Patients with neurological/neurosurgical diseases are treated on another specialized ward as are all patients treated in the surgical departments. The medical team consists of specialists in internal medicine and a specialized intensive care nursing staff. A consultative support is organized in cooperation with the other clinics of the hospital to address systemic problems (eg, the dermatologist for dermatological consultation).

All patients who did not receive CT scan of the head at any time point were excluded as were patients with $\mathrm{CT}$ scan which were not carried out from the ICU, for example, from the emergency ward or neurology department after transfer of a patient. No patients below 18 years were included.

Collected data from medical records included patient characteristics (age, sex, admission diagnosis, underlying disease, comorbidities, days on ICU, death, medication, and laboratory results). Admission diagnoses were combined to generic terms (eg, decompensated cirrhosis and acute hepatitis to "liver failure" or intoxication/unknown cause of vigilance decrease most probably caused by an internal medicine disease to "neurology") as were the underlying diseases (eg, chronic heart failure and coronary heart disease to "cardiology" or cancer diseases to "hemato-oncology"). Anticoagulation was registered as were laboratory results reflecting blood coagulation, normal values for thrombocytes were $>150 / \mathrm{nL}$, Quick $<70 \%$, and partial thromboplastin time $>40$ seconds. It was documented if patients were intubated at the time of CT scan, needed circulatory support (eg, norepinephrine or dobutamine perfusor), and if antibiotics were administered.

\section{Computed Tomography of the Head}

All first CT scans of the head were identified and evaluated retrospectively from the radiology report and physician notes. Second CT scans were evaluated for patients with a negative first CT scan. Computed tomographies were excluded if they were follow-up scans for established diseases.

Indications for CT scan were classified as the most common questions to the radiologist as given from the clinician requesting the CT scan: unconsciousness/syncope, inadequate wake up after end of sedation, seizure, new focal neurology, resuscitation, somnolence/AMS, meningism, clinically suspected cerebral pressure, and others.

New neurological deficits as stated by the treating physician at the time point of CT were determined by reviewing patients charts and classified as follows: anisocoria, aphasia, unconsciousness, tonic and wide pupils, headache, seizure, myoclonus, paresis, vertigo, impaired vision, AMS, and psychic alterations. New findings during HCT (CT-positive group) were classified as all new pathologies found on the CT head scan: abscess, extra-axial bleeding (eg, subdural hematoma), fracture, edema, hydrocephalus, signs of hypoxic brain damage, intracerebral bleeding (ICB)/subarachnoid hemorrhage (SAH), ischemic stroke, tumor, and none. Old findings were classified as all pathological findings which were known before or were classified as chronic changes, not leading to any clinical findings: residual defect (eg, old infarction/bleeding), arteriosclerosis/microvasculature changes, cerebral atrophy, and none. Consequences of HCT were classified as control (direct determination of a repeat scan), immediate surgery, change of therapy (every acute change of treatment directly deducted from the result of the CT scan including do-notresuscitate orders), transfer to another ward/department, and none. If the number of findings was below $10(\mathrm{n}<10)$ and did not fit one of the given categories, it was defined as others, for example, colloidal cysts as a radiologic finding.

\section{Aims of the Study}

The primary aim of this study was the evaluation of the diagnostic yield of head CT scans in a special cohort of patients, namely patients treated on a nontraumatic ICU. Furthermore, outcomes of pathological findings were evaluated. For group comparisons, patients with negative CT scans were compared 
to patients with positive head $\mathrm{CT}$ scans, meaning scans with at least 1 new pathological finding. For survival analysis, patients were divided into survivors and nonsurvivors up to 30 days after the $\mathrm{CT}$ scan. For multivariate analysis and Cox regression, significant findings during group comparisons were included.

\section{Statistical Analysis}

Continuous variables are shown as median and range and categorical variables are reported as frequencies and percentages. Differences between different patient cohorts were determined using the Fisher-Freeman-Halton exact test for categorical variables (McNemar test for dependent variables); for quantitative variables, we used the Mann-Whitney $U$ test or KruskalWallis test (Wilcoxon for dependent variables). For multiple comparisons, Bonferroni correction was applied. Risk factors for positive CT scans were determined using uni- and multivariate binary logistic regression model. For assessment of survival factors, we used a uni- and multivariate Cox regression model. All $P$ values reported are 2 sided. Statistical significance was assumed when the $P$ value was $<.05$. Statistical analyses were performed using SPSS 22 (IBM, Armonk, New York) and Prism 5 (GraphPad Software Inc, San Diego, California).

\section{Results}

\section{Patient Characteristics}

During the given time, 3674 patients were treated on the MICU (around 648 patients/year and 54 patients/month). The initial analysis identified $879(23.92 \%)$ patients who underwent HCT; however, 187 patients were excluded mainly because head CT was carried out before admission or after discharge from ICU. Two patients received head CT for liver transplant evaluation. Finally, 690 patients who underwent head CT scan during the 5 -year time period, which is a rate of $18.8 \%$ of all patients at least receiving $1 \mathrm{CT}$ scan of the head, were evaluated.

Five hundred forty-three (78.7\%) patients had negative CT results, while $147(21.3 \%)$ of all patients had at least 1 new pathological finding. For detailed analysis see Figure 1.

The cohort included 459 (66.5\%) males and 231 (33.5\%) females, median age was 65 (18-92 years). Median time to CT scan was 1 day (0-73), median stay on ICU was 10 days (0-141). Two hundred twenty-eight (33\%) patients of the cohort died on ICU after a median of 9 days (0-125). Patients with a positive CT scan had a significant shorter survival on ICU (5 vs 12 days, $P<.001)$ and a significantly higher death rate $(\mathrm{n}=68,46.3 \%$ vs $\mathrm{n}=160,29.5 \%, P<.001)$.

\section{Comparison of Patients With Positive and Negative CT Scan Results}

Main admission diagnoses were successful resuscitation $(\mathrm{n}=$ $179,25.9 \%)$, followed by pulmonary failure $(\mathrm{n}=124,18 \%)$ and cardiac failure of any kind $(\mathrm{n}=107,15.5 \%)$. There were significantly less positive CTs in patients with admission diagnosis of gastrointestinal bleeding $(0.0 \%$ vs $3.1 \%, P=.03)$,

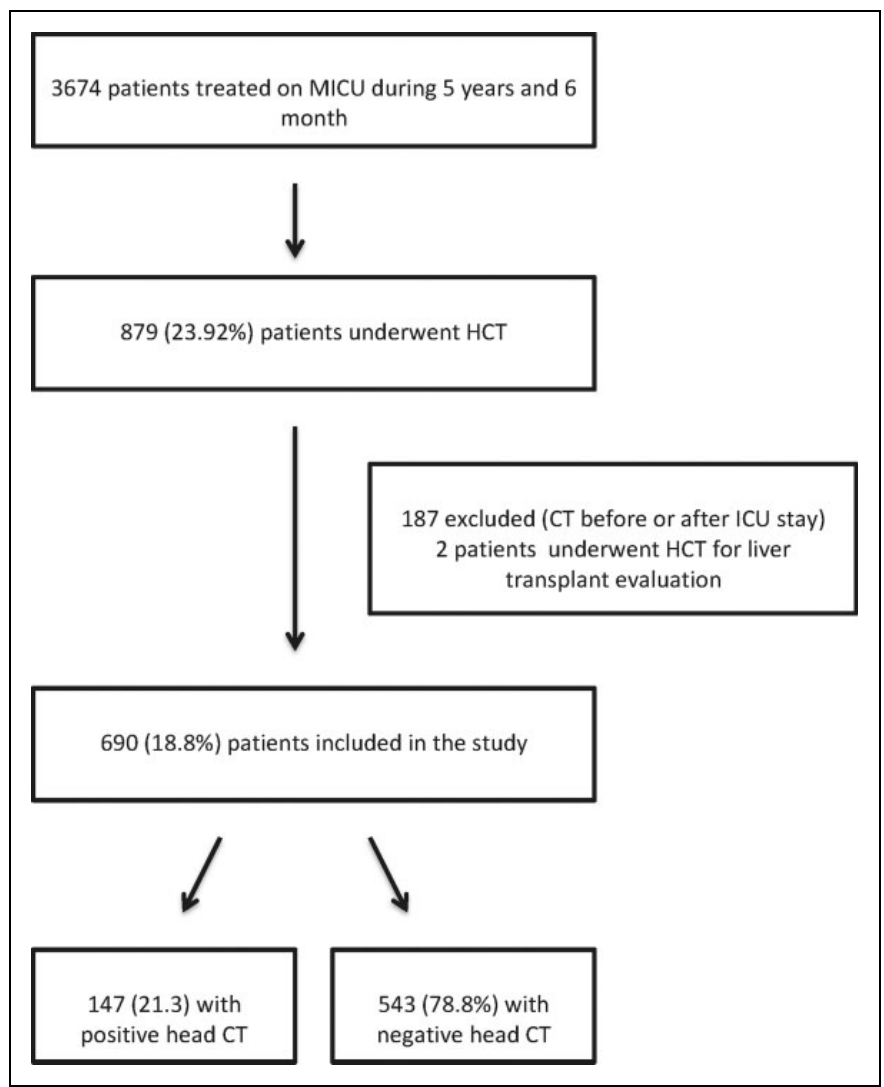

Figure I. Flowchart of included and excluded patients from the MICU and the number of patients with positive and negative head computed tomography $(\mathrm{CT})$ results.

pulmonary failure $(11.6 \%$ vs $19.7 \%, P=.023)$, or sepsis (5.4\% vs $13.3 \%, P=.009$ ), while patients with a neurological diagnosis $(9.5 \%$ vs $4.6 \%, P=.022)$ or resuscitation $(41.5 \%$ vs $21.7 \%, P<.001)$ had significantly more often positive CT scans.

According to comorbidities, namely active tumor disease, atrial fibrillation, and chronic kidney disease, there were no significant differences with $20 \%$ to $30 \%$ in both groups. Three hundred fiftytwo $(51.0 \%)$ patients had at least 1 medication interfering with blood clotting, mainly heparin (prophylactic heparin administration not included; $\mathrm{n}=173,25.1 \%$ ) and combined platelet inhibition (aspirin and P2Y12 inhibitor; $\mathrm{n}=62,9.0 \%$ ). Three hundred ninety-seven $(57.5 \%)$ patients were intubated at the time of CT scan, 289 (41.9\%) needed circulatory support, and $539(78.1 \%)$ received antibiotics. There was no significant difference in laboratory results, namely creatinine, hemoglobin, thrombocytes, prothrombin time, and partial thromboplastin time (data not shown). Significantly more patients in the CT-positive group were intubated $(72.8 \%$ vs $53.4 \%, P<.001)$ and received circulatory support $(50.3 \%$ vs $39.6 \%, P=.009)$.

Overall, we found $4(0.6 \%)$ complications during HCT and transport of these patients to the CT scanner, all were in the CTnegative group. One patient had a seizure during transport, which was treated successfully. One patient had minor allergic reaction to the contrast agent. Two patients had cardiac arrest during transport but were successfully resuscitated after a short time. For detailed data, see Table 1. 
Table I. Patient Characteristics and Admission Diagnoses. ${ }^{\text {a }}$

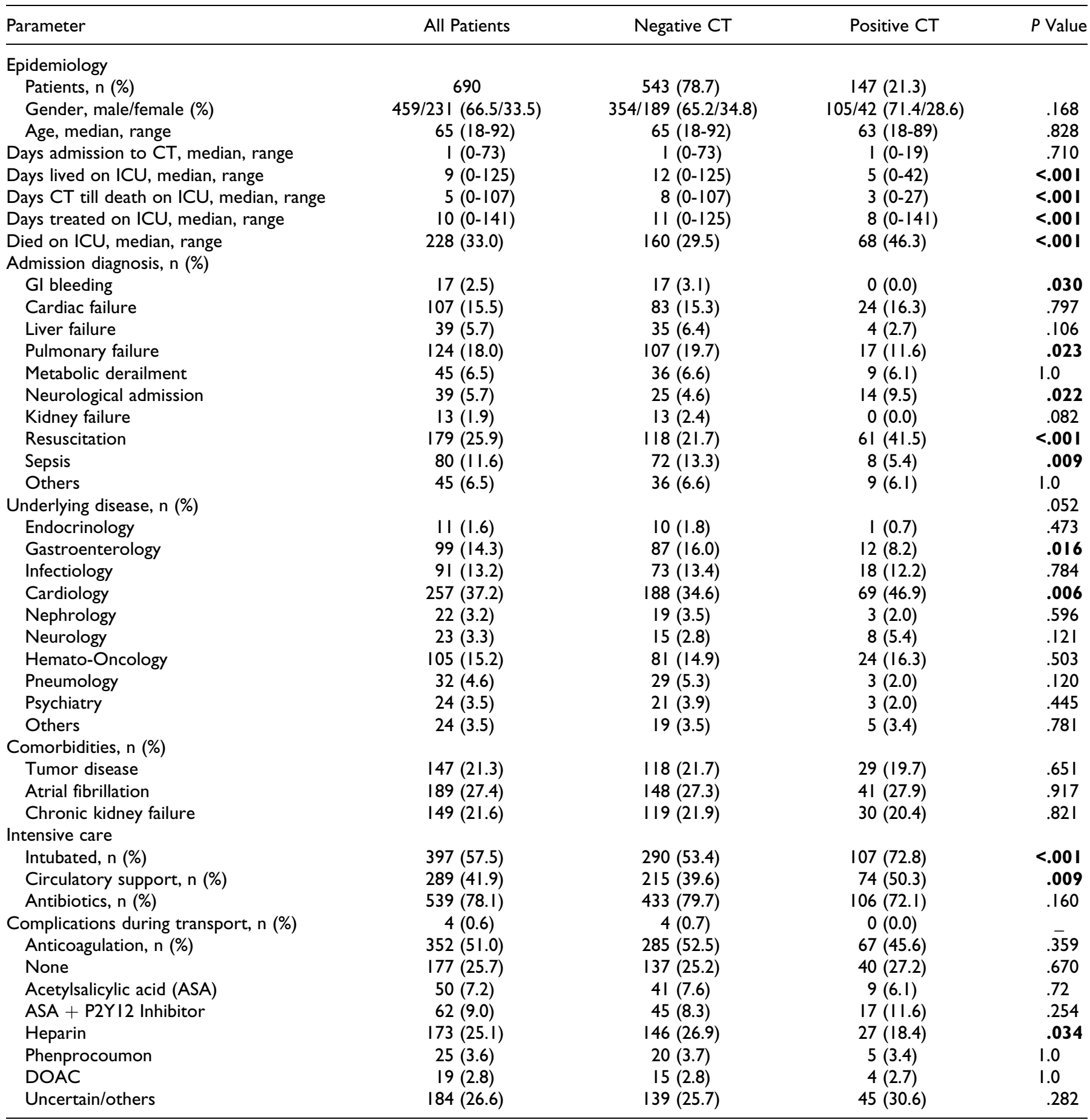

Abbreviations: CT, computed tomography; DOAC, direct oral anticoagulant; GI, gastrointestinal; ICU, intensive care unit.

${ }^{a} V$ ariables are expressed as median and range or as numbers and proportions, as appropriate. All $P$ values reported are 2 sided. Statistical significance was defined as $P \leq .05$.

\section{Main Indications for Acquiring a Head CT}

The main indication for acquiring $\mathrm{CT}$ scan of the head was an AMS in $162(23.5 \%)$ patients, followed by a new focal neurology in $143(20.7 \%)$ patients and an inadequate wake up after stopping sedation in 103 (14.9\%) of all patients. Significantly more patients with a CT scan postresuscitation had a positive finding ( $\mathrm{n}=32,21.8 \%$ vs $\mathrm{n}=56,10.3 \%, P<.001)$, while patients with AMS were significantly less in the positive CT cohort ( $\mathrm{n}=16,10.9 \%$ vs $\mathrm{n}=146,26.9 \%, P<.001)$.

The most common neurologic abnormalities before CT scan were AMS in $185(26.8 \%)$ patients, with significantly less 
Table 2. Indications for CT, Neurologic Deficits, and Pathological Findings. ${ }^{\text {a }}$

\begin{tabular}{|c|c|c|c|c|}
\hline Parameter & All Patients & Negative CT & Positive CT & $P$ Value \\
\hline \multicolumn{5}{|l|}{ Epidemiology } \\
\hline Patients, n (\%) & 690 & $543(78.7)$ & |47 (2|.3) & \\
\hline \multicolumn{5}{|l|}{ Indication for CT, n (\%) } \\
\hline Unconsciousness/syncope & $20(2.9)$ & $17(3.1)$ & $3(2.0)$ & .591 \\
\hline Inadequate wake up & $103(14.9)$ & $74(13.6)$ & $29(19.7)$ & .069 \\
\hline Resuscitation & $88(12.8)$ & $56(10.3)$ & $32(21.8)$ & $<.001$ \\
\hline AMS & $162(23.5)$ & $146(26.9)$ & $16(10.9)$ & $<.001$ \\
\hline Others & $114(16.5)$ & $93(17.1)$ & $21(14.3)$ & .454 \\
\hline \multicolumn{5}{|l|}{ Neurologic deficit, n (\%) } \\
\hline None & $38(5.5)$ & $34(6.3)$ & $4(2.7)$ & .105 \\
\hline Anisocoria & $104(15.1)$ & $80(14.7)$ & $24(16.3)$ & .606 \\
\hline Myoclonus & $31(4.5)$ & $19(3.5)$ & $12(8.2)$ & .023 \\
\hline Paresis & $27(3.9)$ & $14(2.6)$ & $13(8.8)$ & .001 \\
\hline Psychic alteration & $51(7.4)$ & $49(9.0)$ & $2(1.4)$ & $<.001$ \\
\hline AMS & $185(26.8)$ & $160(29.5)$ & $25(17.0)$ & .002 \\
\hline Others & $4 I(5.9)$ & $34(6.3)$ & $7(4.8)$ & .562 \\
\hline \multicolumn{5}{|l|}{ Old findings, n (\%) } \\
\hline None & $477(69.1)$ & $383(70.5)$ & $94(63.9)$ & .132 \\
\hline Residual defect (infarct/bleeding) & $120(17.4)$ & $78(14.4)$ & $42(28.6)$ & $<.001$ \\
\hline Arteriosclerosis/microvasculatory changes & $39(5.7)$ & $36(6.6)$ & $3(2.0)$ & .042 \\
\hline Cerebral atrophy & $16(2.3)$ & $15(2.8)$ & I (0.7) & .215 \\
\hline Others & $36(5.2)$ & $30(3.5)$ & $6(4.1)$ & .676 \\
\hline
\end{tabular}

Abbreviations: AMS, altered mental state; CT, computed tomography; ICU, intensive care unit; $\mathrm{GI}$, gastrointestinal.

${ }^{a}$ Variables are expressed as median and range or as numbers and proportions, as appropriate, and all $P$ values reported are 2 sided. Statistical significance was defined as $P \leq .05$.

patients in the CT-positive cohort $(\mathrm{n}=25,17 \%$ vs $\mathrm{n}=160$, $29.5 \%, P=.002)$. Significantly more patients in the CT-positive group had the finding of wide pupils on both sides (4.1\% vs $1.1 \%, P=.025)$, myoclonus ( $8.2 \%$ vs $3.5 \%$, $P=.023)$, and new paresis $(8.8 \%$ vs $2.6 \%, P=.001)$, while the finding of psychic alteration was significantly less in CT-positive patients $(1.4 \%$ vs $9.0 \%, P=.002)$.

One hundred fifty-eight $(22.9 \%)$ patients underwent contrast-enhanced CT scan without any difference between both groups. The most common question to the radiologist was for the diagnosis of a new ICB in 392 (56.8\%) of all patients, which led to a new pathological finding in significantly less patients ( $\mathrm{n}=67,45.6 \%$ vs $\mathrm{n}=325,59.9 \%, P=.003)$, while the question for hypoxic brain damage in 102 (14.8\%) patients led to a new pathological finding in significantly more patients ( $\mathrm{n}=41,27.9 \%$ vs $\mathrm{n}=61,11.2 \%, P<.001$; data not shown). For detailed analysis, see Tables 1 and 2 .

\section{Acute Changes on Head CTs and the Clinical Consequences}

Five hundred forty-three $(78.7 \%)$ of all patients had a negative CT scan, while 147 (21.3\%) had a new pathological finding. The most common new finding was ICB of any kind (intracerebral hemorrhage, subdural hemorrhage, and SAH) in 44 (29.9\%) patients, followed by signs of hypoxic brain damage in $30(20.4 \%)$ patients and new ischemic stroke in $36(24.5 \%)$ patients. For detailed analysis, see Figure 2.

Several findings in the CT scans were old or incidental findings such as residual defects (old infarction/bleeding) in $120(17.4 \%)$ patients, with higher rates in the CT-positive group ( $\mathrm{n}=42,28.6 \%$ vs $\mathrm{n}=78,14.4 \%, P<.001$ ), or signs of microvasculatory changes/arteriosclerosis in $5.7 \%$ of all patients. However, 477 (69.1\%) patients showed no pathological alterations of any kind. 


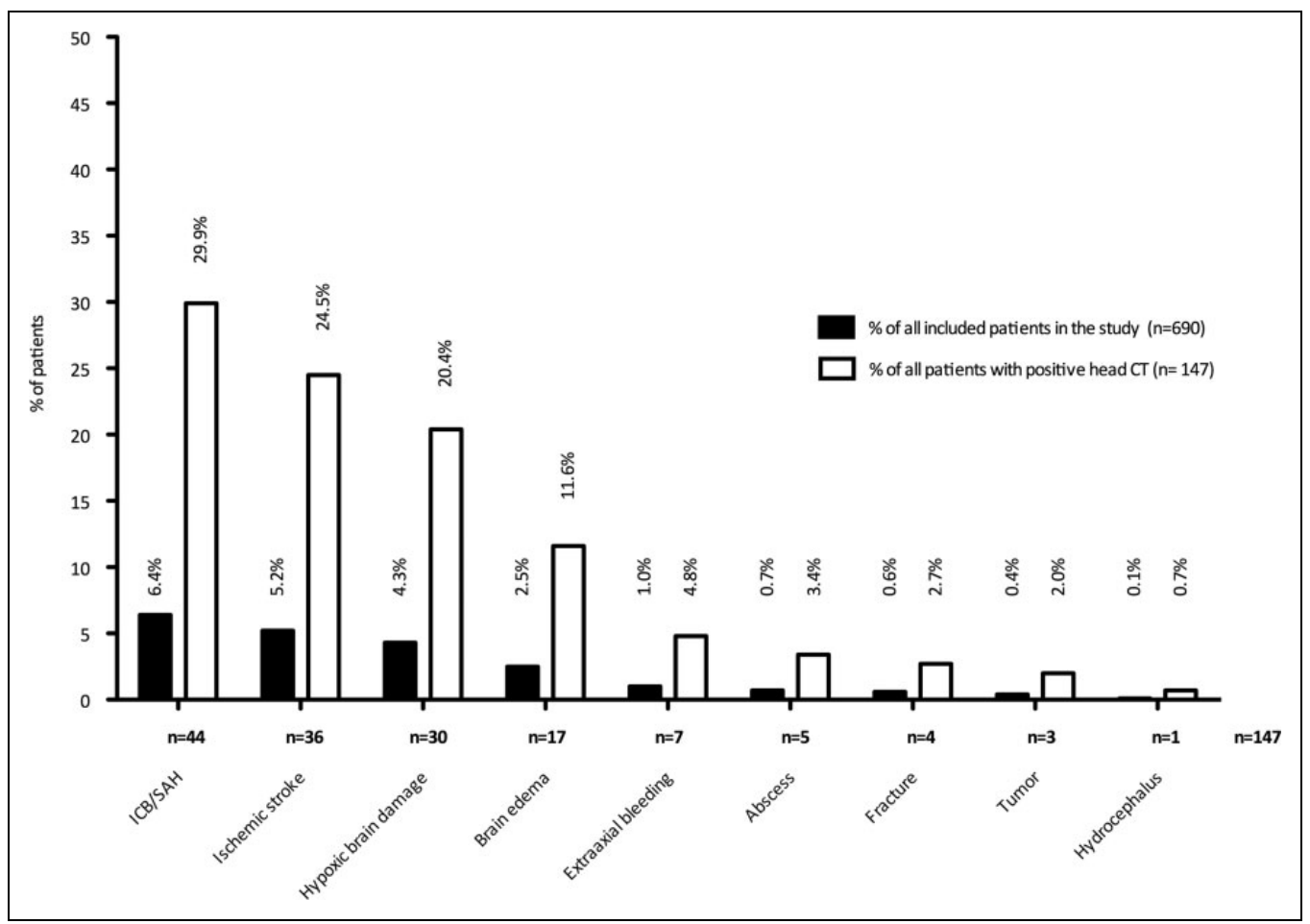

Figure 2. Bar chart with new pathological findings of the cerebral computed tomography (CT) scans as part of the whole patient cohort (black bars, $n=690$ patients) and the patients with a positive head CT (white bars, $n=147$ patients).

In 460 (66.7\%) patients, the CT scan had no obvious clinical consequences, however, $80.5 \%(\mathrm{n}=437)$ were in the CTnegative group, while only $15.6 \%(\mathrm{n}=23)$ were in the CTpositive group $(P<.001)$. In $46(6.7 \%)$ patients, the CT scan itself led to a change of therapy of any kind, $0.9 \%(\mathrm{n}=5)$ in the CT-negative group and $27.9 \%(\mathrm{n}=41)$ in the CT-positive group $(P<.001) ; 2.3 \%$ of all patients $(\mathrm{n}=16)$ were transferred to another ward with significantly more patients in the CTpositive group $(\mathrm{n}=9,6.1 \%$ vs $\mathrm{n}=7,1.3 \%, P=.002)$. Five (3.4\%) patients in the CT-positive group underwent direct surgery. For $22.0 \%$ of all patients $(\mathrm{n}=152)$, a second control CT was planned due to the findings in the first CT. For detailed analysis, see Table 2 .

\section{Diagnostic Yield of a Repeated Head CT}

One hundred eight (15.7\%) of all patients with a negative first CT scan underwent a second CT during the ICU stay. Thirtysix $(33.3 \%)$ patients showed new pathological findings in a second HCT. The second CT was done median 5 days (0-34) after the first. The indications and neurologic abnormalities did not differ significantly between the first and second CT scan (data not shown). Most common new pathological finding was hypoxic brain damage in $17(15.7 \%)$ patients and ischemic stroke in $8(7.4 \%)$ patients. As a consequence, $27(25.0 \%)$ patients underwent another control CT, the therapy was changed for $15(13.9 \%)$ patients, and $6(5.6 \%)$ patients were transferred to another ward.
Fourteen of 17 patients with hypoxic brain damage diagnosed in the second HCT (median day 4, range 2-8) were admitted postresuscitation with negative findings in an early HCT (day 0-1). For detailed analysis, see Table 3.

\section{Risk Factors Associated With Acute Pathological Findings on Head CT}

We aimed at finding risk factors associated with new pathological findings in head CT scans. Therefore, we first conducted an univariate analysis including all factors which probably could influence the variable positive head CT such as age, gender, the admission diagnosis, anticoagulation, and neurologic abnormalities. In univariate analysis, we could identify several factors increasing the odds of a positive head CT as admission after resuscitation (odds ratio [OR]: 2.56, 1.74-3.76, $P<.001)$ or admission with unidentified neurologic abnormalities (OR: 2.18, 1.10-4.31, $P=.025$ ), intubation (OR: 2.32, 1.56-3.47, $P<.001$ ), circulatory support (OR: 1.66, 1.14-2.41, $P=.008)$, or manifest neurological deficits as, for example, new paresis (OR: 3.67, 1.68-7.98, $P=.001$ ). However, other factors in this cohort seem to come along with a decreased risk of a positive head CT as there are the admission with pulmonary failure (OR: $0.51,0.29-0.89, P=.018$ ) or sepsis (OR: $0.38,0.18-0.80, P=.011)$ or the neurologic abnormalities such as psychic alteration (OR: 0.14, 0.03-0.58) and AMS (OR: $0.5,0.31-0.79, P=.003$ ). After inclusion of all the factors in a multivariate model, 5 independent predictors for 
Table 3. Results of Second CT Scans. ${ }^{a}$

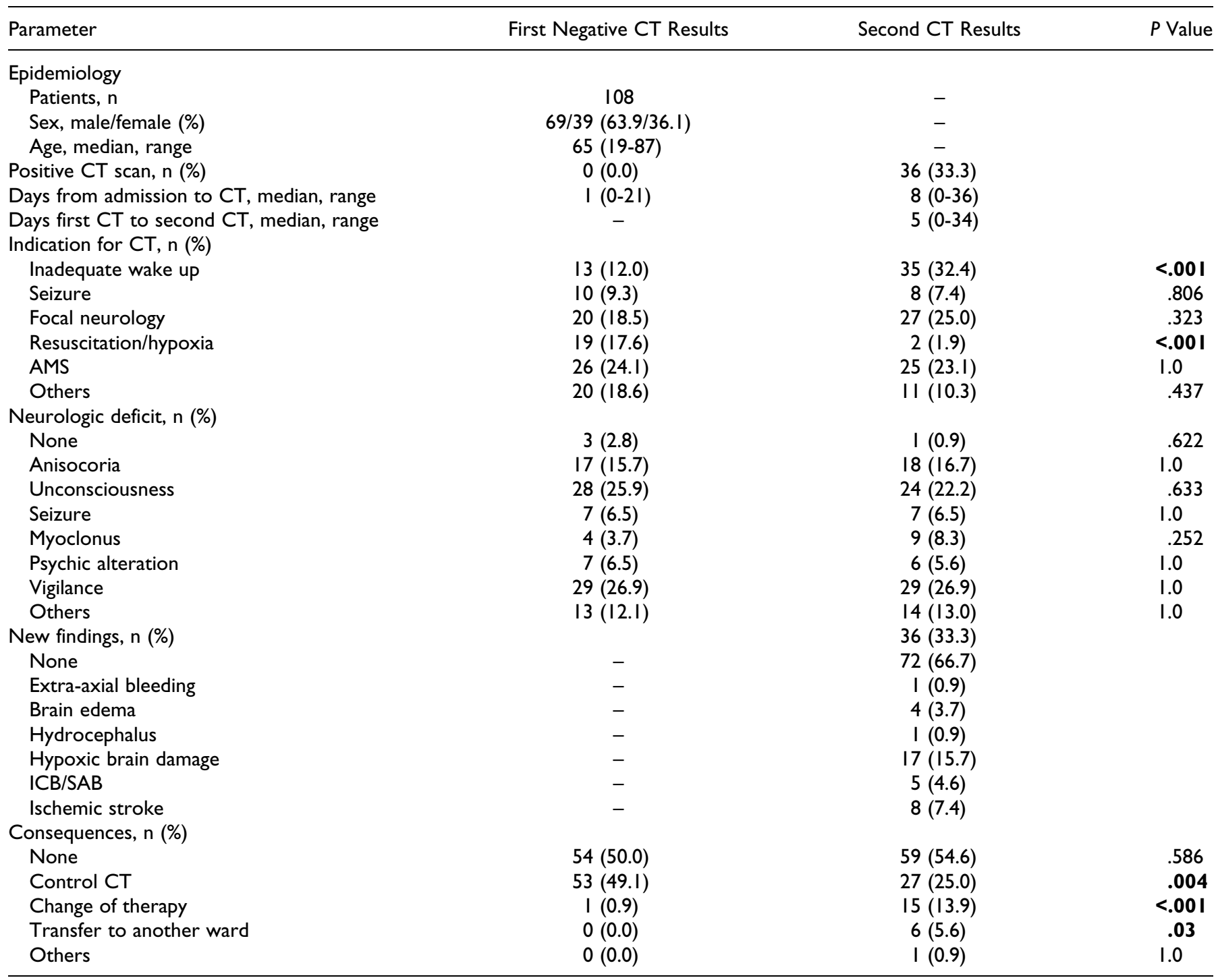

Abbreviations: CT, computed tomography; GI, gastrointestinal; ICB, intracerebral bleeding; ICU, intensive care unit; SAB, subarachnoid hemorrhage.

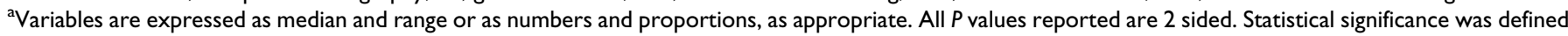
as $P \leq .05$.

a positive CT scan remained, the admission after resuscitation (OR: $2.71,1.78-4.13, P<.001)$ and the admission with neurologic abnormalities (OR: 3.21, 1.57-6.60, $P=.001$ ), furthermore, the diagnosis of a new paresis (OR: 4.12, 1.829.32, $P=.001)$.

Psychic alteration and AMS were both independent predictors of a reduced risk of positive head CT (OR: 0.18, 0.04-0.77, $P=.021$ and $0.55,0.34-0.9, P=.016$, respectively). For detailed analysis, see Table 4.

\section{Factors Associated With Decreased Survival in Patients Undergoing CT Scan of the Head}

For the evaluation of factors predicting decreased survival in this selected cohort, we evaluated the 30-day mortality after the first HCT scan. During the given time frame, 256 (37.1\%) patients died. While $31.6 \%(\mathrm{n}=81)$ had a positive CT scan in the nonsurvivor group, only $15.2 \%(n=66)$ could be identified in the survivor group. Significantly more patients with the admission diagnosis of resuscitation were in the nonsurvivor group ( $\mathrm{n}=97,37.6 \%$ vs $\mathrm{n}=82,18.9 \%, P<.001)$, while more patients with cardiac failure were in the survivor group (n $=84,19.4 \%$ vs n $=23,9.0 \%, P<.001)$. To evaluate whether a positive CT scan during MICU stay is an independent predictor of death, we again conducted uni- and multivariate Cox regression. While several factors were associated with death in univariate analysis, a positive CT scan remained an independent predictor of death (hazard ratio [HR]: 2.05, 156-2.7, $P<.001)$ along with the admission diagnosis of gastroenterological disease (HR: 2.16, 1.05-4.44, $P=.037$ ), resuscitation (HR: 2.22, 1.69-2.94, $P<.001$ ), sepsis (HR: 1.55, 1.04-2.30, $P=.031$ ), diagnosed tumor disease (HR: 1.35, 1.01-1.82, 
Table 4. Univariate and Multivariate Analyses for Patient Factors Significantly Associated With Positive CT Scan. ${ }^{\text {a }}$

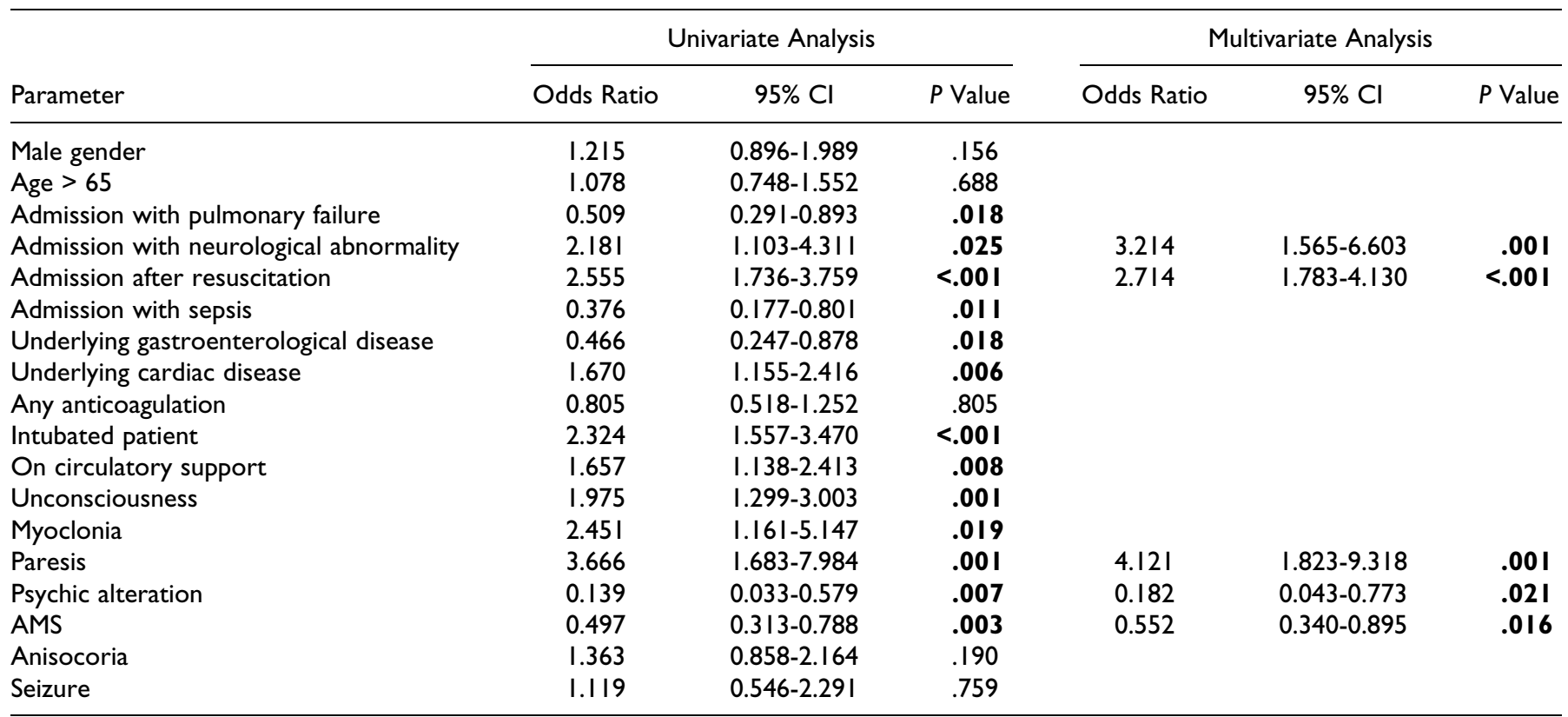

Abbreviations: AMS, altered mental state; $\mathrm{Cl}$, confidence interval; $\mathrm{HR}$, hazard ratio.

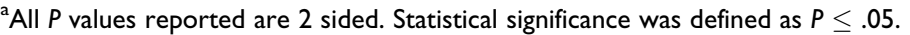

Table 5. Univariate and Multivariate Analyses of Parameters Associated With 30-Day Survival Post-CT Scan. ${ }^{\text {a }}$

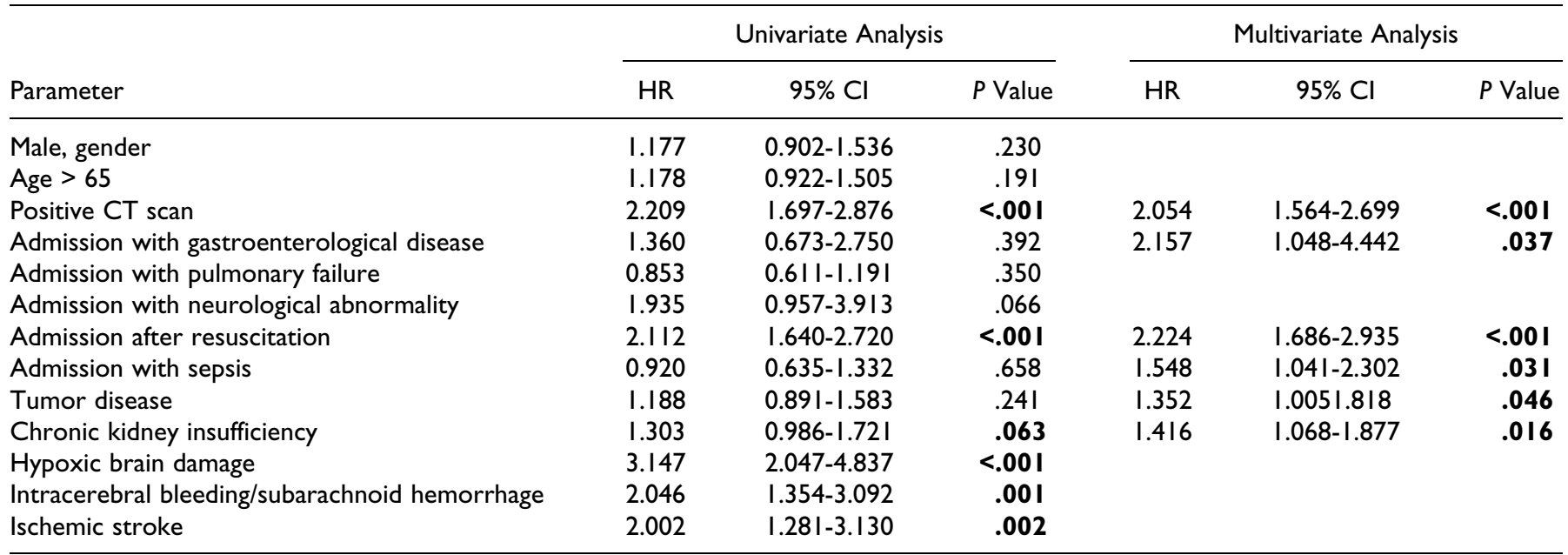

Abbreviations: $\mathrm{Cl}$, confidence interval; $\mathrm{CT}$, computed tomography; $\mathrm{HR}$, hazard ratio.

${ }^{\text {a All }} P$ values reported are 2 sided. Statistical significance was defined as $P \leq .05$.

$P=.046$ ), and chronic kidney insufficiency (HR: 1.42, $1.07-1.88, P=.016)$ in this cohort. For detailed analysis, see Tables 5 and 6.

\section{Discussion}

Data about the diagnostic yield of head CTs for patients treated on MICU are scarce; to our knowledge, this is the largest study so far evaluating HCTs from exclusively MICU patients.

In our study, $78.7 \%$ had negative CT results, while $21.3 \%$ of all patients had at least 1 new pathological finding. The main indication for acquiring CT scan of the head was an AMS in $23.5 \%$ of all patients. The most common new finding was ICB in $6.4 \%$. In $6.7 \%$, the CT scan itself led to a change of therapy of any kind in patients with positive HCT. Admission after resuscitation or a new focal neurology were independent predictors of a positive CT, while psychic alteration and AMS were both independent predictors of a higher chance of a negative head CT.

Neurological dysfunction is a common finding even in nonneurological patients on $\mathrm{MICU},{ }^{23}$ and especially delirium, psychic alterations, and AMS may occur very frequently on 
Table 6. 30-Day Mortality After CT Scan of All Patients. ${ }^{\text {a }}$

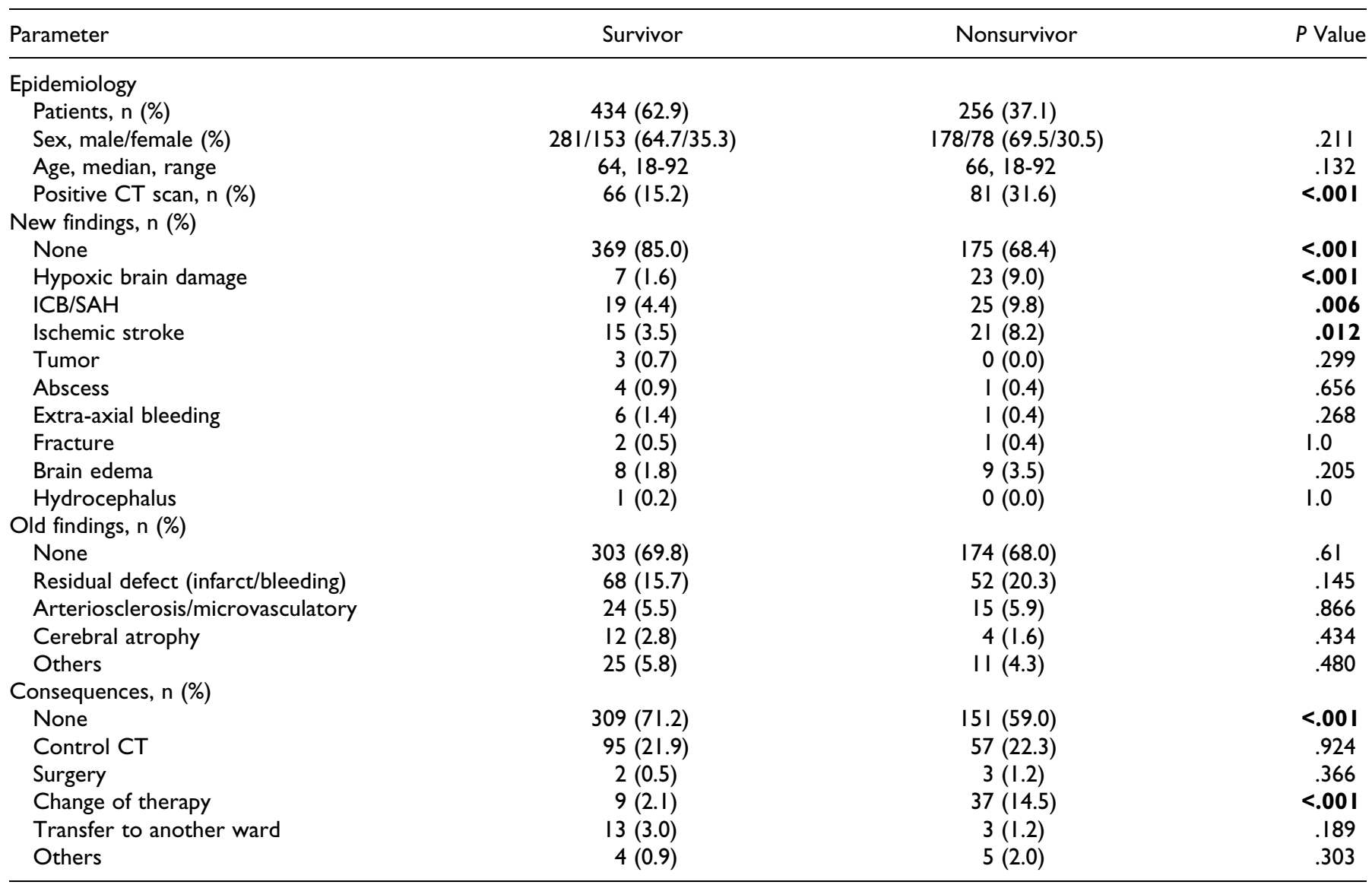

Abbreviations: CT, computed tomography; DOAC, direct oral anticoagulant; GI, gastrointestinal; ICB, intracerebral bleeding; ICU, intensive care unit; SAB, subarachnoid hemorrhage.

${ }^{a}$ Variables are expressed as median and range or as numbers and proportions, as appropriate. All $P$ values reported are 2 sided. Statistical significance was defined as $P \leq .05$.

MICU. ${ }^{24-26}$ In 2000, Rafanan et al conducted a study comparable to ours reviewing $230 \mathrm{MICU}$ patients undergoing HCT being $21 \%$ of all patients. Most of the HCTs were done due to AMS (88\%). Thirty-seven percent of all HCTs showed a positive finding. A new neurological deficit was identified as the only independent risk factor for a positive CT, however, the authors concluded that there is no reliable predictor for the clinician. ${ }^{20}$ Salerno and colleagues identified 123 (16.6\%) patients with HCT during a 2 -year period. Twenty-six (21.1\%) patients showed a new pathological finding, but these patients were preselected for neurological abnormalities. The most common pathological finding was an ischemic stroke (49\%) comparable to the study from Rafanan and colleagues. Patients with positive HCT had more comorbidities and more often had vasopressors as a medication, but a predictive risk factor could not be identified. Another study evaluated 42 patients who remained unresponsive after cessation of sedation for 48 hours without any known neurological disease. ${ }^{19}$ Interestingly, only 1 patient was diagnosed with a small SAH, all other HCTs were nondiagnostical, $60 \%$ had a normal HCT, and the rest were incidental findings not explaining the patients' clinic. The authors concluded that HCT in these patients is of low yield as it did not change the therapeutic strategies and 90\% regained consciousness later on.

In 2012, Purmer et al prospectively evaluated head CT scans on 2 mixed ICUs including neurosurgical patients. Only 72 medical patients were included, with $39 \%$ positive HCTs compared to $57 \%$ in surgical patients. The main reason for HCT was to exclude cerebral hemorrhage in more than $50 \%$ of all patients, however, was diagnosed in only 2 patients. In $57 \%$ of all patients, the CT did not lead to any treatment change. ${ }^{18}$ In 2014, Khan et al conducted a retrospective study from 3 ICUs (surgical and medical) and evaluated 706 patients: $17.2 \%$ had a focal neurology, $11.0 \%$ a seizure, and $70.5 \%$ an AMS; $12.1 \%$ showed acute changes on HCT. The highest rate of positive scans was among patients with neurologic deficits (30\%) but was low in AMS patients (7.4\%). Interestingly, sepsis decreased the odds for a positive head $\mathrm{CT}$, comparable to our results. ${ }^{22}$ Patients postresuscitation and with severe liver disease were excluded in this study.

Most of these data are comparable to ours, and the rate of around $20 \%$ positive CTs in the given cohorts does not seem to differ by a wide range. Like in our study, a new focal neurology seems to be a very good predictor for a positive 
CT, while especially an AMS seems to be a bad predictor for positive HCT.

Some admission diagnoses seem to come along with a higher chance of a positive CT, others tend to yield a very low rate. Interestingly, in patients with a gastroenterological admission diagnosis (GI bleeding), there were significantly more patients with negative CT scans than positive. Often patients with severe liver disease present with AMS and coagulopathy or thrombocytopathy at the same time, both factors increasing the physician's sensitivity for performing HCT. Rahimi and Rockey analyzed patients with cirrhosis and AMS undergoing HCT. More than two-third underwent HCT and no pathological findings were diagnosed, while all patients with a new pathological finding had a distinct focal neurology at the same time. ${ }^{27}$ The idea that patients with cirrhosis are at an increased risk of ICB cannot be hold up as well, for example, Lai et al found nearly the same risk in cirrhotic and noncirrhotic patients during a long-time follow-up, ${ }^{28}$ which is comparable to other publications. ${ }^{29}$ Furthermore, CT scans in our cohort tended to yield significantly less positive findings in patients with pulmonary failure and sepsis probably pointing out a $\mathrm{CT}$ overuse in these patients. It is well established that severe illness like pneumonia and especially sepsis can cause mental alterations of any kind. Sepsis-associated encephalopathy can cause alterations of consciousness, ranging from delirium to coma, seizure, or focal neurological signs, however, pathological findings in HCT scans will be quite rare and magnetic resonance imaging is considered the best option when imaging analysis is warranted in these patients. ${ }^{30,31}$

In our cohort, we could not identify anticoagulants or impaired coagulation as a risk factor for a positive HCT. Patients are preselected for MICU, however, patients with intracerebral hemorrhage, including those caused by anticoagulation, may mainly be admitted to neurological/ neurosurgical care. Furthermore, the risks of anticoagulants (phenprocoumon and direct oral anticoagulants) for bleeding complications are well known and quite low; therefore, patient number may not be high enough. ${ }^{32,33}$ Thrombocytopenia by any cause is known to be an independent risk factor for bleeding, however, is a very rare event, for example, for immune thrombocytopenia (ITP) at around $1 \%$ for chronic ITP patients with very low platelets. ${ }^{34,35}$

Resuscitation was an independent risk factor for a positive $\mathrm{CT}$ in this cohort. The role of postresuscitation HCT is still not clear, and it can be useful early after resuscitation for diagnosis of the cause for cardiac arrest and later for adding information to prognosis of the patient. ${ }^{36,37}$ In our cohort, we identified 9 patients with SAH as cause of cardiac arrest who were only diagnosed because of an early HCT. Furthermore, 14 patients with no pathologies in the first HCT showed hypoxic brain damage during a later one. The right time point of HCT in these patients depends on the indication, and studies on this topic are scarce. ${ }^{38-40}$ Out-of-hospital cardiac arrest due to cerebrovascular events is not a rare event, for example, in 1 study, SAH was the cause in up to $2 \%$ in an unselected cohort. ${ }^{41}$ Furthermore, even cardiac arrest caused by cerebral hemorrhage often comes along with abnormalities in electrocardiogram or even ultrasound of the heart. ${ }^{42}$ Signs of ischemic brain damage in CT can occur quite early, however, are not sufficient for prognosis at this time point. ${ }^{36,38,40}$ Current American Heart Association guidelines suggest a HCT for acquiring a prognosis of the patient not earlier than 24 hours. ${ }^{36}$ Cardiac arrest caused by different kinds of brain damage is not an uncommon event and therapy would be changed completely for these patients, early HCT after hospital admission should be considered for every single patient. $22.9 \%$ of all HCTs were carried out with contrast agent enhancement, mostly for specific questions (HIV patients with suspected toxoplasmosis, central nervous system lymphoma), however, no difference in both groups was detected. Two patients had an allergic reaction, therefore, the use of contrast agents in HCT should be evaluated carefully and discussed with the radiologist before.

A review of the literature about intrahospital transport showed that diagnostic procedures acquired via transport lead to a change in patient management in $40 \%$ to $50 \%{ }^{43}$ This is comparable to our findings, where overall $66.7 \%$ of all patients after HCT did not undergo any direct change of treatment. A positive CT scan let so a significant change in $37.4 \%$ of patients and a control CT in $43.5 \%$. The rate of complications during transport in our cohort was very low with $4(0.6 \%)$ patients. This is even very low compared to published data, ${ }^{6}$ however, we only included severe complications, none of it was fatal. So the risk of transport, at least in our cohort, seems neglectable.

Several limitations have to be addressed. As this is a retrospective study, all data were drawn from patient's files, which could have led to missed or incomplete information; furthermore, not every patient underwent HCT, which could lead to confounding by the indications. All patients were treated at a single tertiary center, therefore, could not represent nationwide practice and transferability to other ICUs. The severity of the abnormalities found on HCTs was not specifically addressed. As we included patients after successful resuscitation and these patients tend to have a bad survival rate and higher probability to find pathological changes in HCT, this may be a bias.

\section{Conclusions}

Taken together we could show that HCT in patients with AMS or psychic alterations is of quite low diagnostic yield. Especially in patients with sepsis with AMS, CT scans of the head seem to be overused. Furthermore, deteriorated coagulation, even in patients with severe liver disease, seems not to be such a strong risk factor for intracerebral pathologies as often assumed by clinicians. Computed tomography scans directly after successful resuscitation should be evaluated for every single patient and any new neurological deterioration should be further investigated by HCT.

\section{Authors' Note}

Fabian Finkelmeier, Sophie Walter, and Harald Farnik designed the study. Fabian Finkelmeier, Sophie Walter, Anjali Cremer, Andrea Tal, Kai-Henrik Peiffer, Thomas Vogl, and Harald Farnik collected data. 
Fabian Finkelmeier, Sophie Walter, Thomas Vogl, Stephan Fichtlscherer, Mireen Friedrich-Rust, Jörg Bojunga, and Harald Farnik F analyzed the data. Fabian Finkelmeier, Thomas Vogl, Stephan Fichtlscherer, Mireen Friedrich-Rust, Jörg Bojunga, and Harald Farnik drafted the article. Thomas Vogl, Stefan Zeuzem, Stephan Fichtlscherer, Mireen Friedrich-Rust, and Jörg Bojunga critically revised the article.

\section{Acknowledgments}

The authors gratefully acknowledge the work of the staff of the critical care unit and the staff of the radiology unit.

\section{Declaration of Conflicting Interests}

The author(s) declared no potential conflicts of interest with respect to the research, authorship, and/or publication of this article.

\section{Funding}

The author(s) received no financial support for the research, authorship, and/or publication of this article.

\section{References}

1. Brenner DJ, Hall EJ. Computed tomography-an increasing source of radiation exposure. $N$ Engl J Med. 2007;357(22): 2277-2284. doi:10.1056/NEJMra072149.

2. Lell MM, Wildberger JE, Alkadhi H, Damilakis J, Kachelriess M. Evolution in computed tomography: the battle for speed and dose. Invest Radiol. 2015;50(9):629-644. doi:10.1097/RLI. 0000000000000172 .

3. Berrington de González A, Mahesh MS, Kim KP, et al. Projected cancer risks from computed tomographic scans performed in the United States in 2007. Arch Intern Med. 2009;169(22): 2071-2077. doi:10.1001/archinternmed.2009.440.

4. Smith-Bindman R, Lipson J, Marcus R, et al. Radiation dose associated with common computed tomography examinations and the associated lifetime attributable risk of cancer. Arch Intern Med. 2009;169(22):2078-2086. doi:10.1001/archinternmed. 2009.427.

5. Lahner D, Nikolic A, Marhofer P, et al. Incidence of complications in intrahospital transport of critically ill patients-experience in an Austrian university hospital. Wien Klin Wochenschr. 2007;119(13-14):412-416. doi:10.1007/s00508-007-0813-4.

6. Fanara B, Manzon C, Barbot O, Desmettre T, Capellier G. Recommendations for the intra-hospital transport of critically ill patients. Crit Care. 2010;14(3):R87. doi:10.1186/cc9018.

7. Emanuel E, Tanden N, Altman S, et al. A systemic approach to containing health care spending. $N$ Engl J Med. 2012;367(10): 949-954. doi:10.1056/NEJMsb1205901.

8. Langkjær S, Hassager C, Kjaergaard J, et al. Prognostic value of reduced discrimination and oedema on cerebral computed tomography in a daily clinical cohort of out-of-hospital cardiac arrest patients. Resuscitation. 2015;92:141-147. doi:10.1016/j.resuscitation.2015.03.023.

9. Giglio P, Bednarczyk EM, Weiss K, Bakshi R. Syncope and head CT scans in the emergency department. Emerg Radiol. 2005; 12(1-2):44-46. doi:10.1007/s10140-005-0434-z.
10. Mitsunaga MM, Yoon HC. Head CT Scans in the emergency department for syncope and dizziness. AJR Am J Roentgenol. 2015;204(1):24-28. doi:10.2214/AJR.14.12993.

11. Grossman SA, Fischer C, Bar JL, et al. The yield of head CT in syncope: a pilot study. Intern Emerg Med. 2007;2(1):46-49. doi: 10.1007/s11739-007-0010-5.

12. Pires LA, Ganji JR, Jarandila R, Steele R. Diagnostic patterns and temporal trends in the evaluation of adult patients hospitalized with syncope. Arch Intern Med. 2001;161(15):1889-1895.

13. Stiell IG, Wells GA, Vandemheen K, et al. The Canadian CT Head Rule for patients with minor head injury. Lancet. 2001; 357(9266):1391-1396. doi:10.1016/S0140-6736(00)04561-X.

14. Easter JS, Grossman SA, Woodruff MM, Rosen CL. When the rules do not work: head injury without loss of consciousness. $J$ Emerg Med. 2008;35(1):77-80. doi:10.1016/j.jemermed. 2008.04.002.

15. Haydel MJ, Preston CA, Mills TJ, Luber S, Blaudeau E, DeBlieux P. Indications for computed tomography in patients with minor head injury. N Engl J Med. 2000;343(2):100-105.

16. Bent C, Lee PS, Shen PY, Bang H, Bobinski M. Clinical scoring system may improve yield of head CT of non-trauma emergency department patients. Emerg Radiol. 2015;22(5):511-516. doi:10. 1007/s10140-015-1305-x.

17. Owlia M, Yu L, Deible C, Hughes MA, Jovin F, Bump GM. Head CT scan overuse in frequently admitted medical patients. Am J Med. 2014;127(5):406-410. doi:10.1016/j.amjmed.2014.01.023.

18. Purmer IM, van Iperen EP, Beenen LFM, et al. Brain computer tomography in critically ill patients-a prospective cohort study. BMC Med Imaging. 2012;12(1):34. doi:10. 1186/1471-2342-12-34.

19. Balachandran JS, Jaleel M, Jain M, et al. Head CT is of limited diagnostic value in critically ill patients who remain unresponsive after discontinuation of sedation. BMC Anesthesiol. 2009;9:3. doi: 10.1186/1471-2253-9-3.

20. Rafanan AL, Kakulavar P, Perl J, Andrefsky JC, Nelson DR, Arroliga AC. Head computed tomography in medical intensive care unit patients: clinical indications. Crit Care Med. 2000; 28(5):1306-1309.

21. Salerno D, Marik PE, Daskalakis C, Kolm P, Leone F. The role of head computer tomographic scans on the management of MICU patients with neurological dysfunction. J Intensive Care Med. 2009;24(6):372-375. doi:10.1177/0885066609344940.

22. Khan S, Guerra C, Khandji A, Bauer RM, Claassen J, Wunsch H. Frequency of acute changes found on head computed tomographies in critically ill patients: a retrospective cohort study. J Crit Care. 2014;29(5):884.e7-884.e12. doi:10.1016/j.jcrc. 2014.05.001.

23. Stevens RD, Nyquist PA. Coma, delirium, and cognitive dysfunction in critical illness. Crit Care Clin. 2006;22(4):787-804. doi: 10.1016/j.ccc.2006.11.006.

24. Devlin JW, Fong JJ, Fraser GL, Riker RR. Delirium assessment in the critically ill. Intensive Care Med. 2007;33(6):929-940. doi:10. 1007/s00134-007-0603-5.

25. Dubois MJ, Bergeron N, Dumont M, Dial S, Skrobik Y. Delirium in an intensive care unit: a study of risk factors. Intensive Care Med. 2001;27(8):1297-1304. 
26. Girard TD, Pandharipande PP, Ely EW. Delirium in the intensive care unit. Crit Care. 2008;12(suppl 3):S3. doi:10.1186/ cc6149.

27. Rahimi RS, Rockey DC. Overuse of head computed tomography in cirrhosis with altered mental status. Am J Med Sci. 2016; 351(5):459-466. doi:10.1016/j.amjms.2016.02.022.

28. Lai $\mathrm{CH}$, Cheng PY, Chen YY. Liver cirrhosis and risk of intracerebral hemorrhage: a 9-year follow-up study. Stroke. 2011;42(9):2615-2617. doi:10.1161/STROKEAHA.111. 617076.

29. Donovan LM, Kress WL, Strnad LC, et al. Low likelihood of intracranial hemorrhage in patients with cirrhosis and altered mental status. Clin Gastroenterol Hepatol. 2015;13(1):165-169. doi:10.1016/j.cgh.2014.05.022.

30. Adam N, Kandelman S, Mantz J, Chrétien F, Sharshar T. Sepsisinduced brain dysfunction. Expert Rev Anti Infect Ther. 2013; 11(2):211-221. doi:10.1586/eri.12.159.

31. Tsuruta R, Oda Y. A clinical perspective of sepsis-associated delirium. J Intensive Care. 2016;4:18. doi:10.1186/s40560-0160145-4.

32. Adam SS, McDuffie JR, Ortel TL, Williams JW. Comparative effectiveness of warfarin and new oral anticoagulants for the management of atrial fibrillation and venous thromboembolism: a systematic review. Ann Intern Med. 2012; 157(11):796-807. doi:10.7326/0003-4819-157-10-20121120000532.

33. Shoeb M, Fang MC. Assessing bleeding risk in patients taking anticoagulants. J Thromb Thrombolysis. 2013;35(3):312-319. doi: 10.1007/s11239-013-0899-7.

34. Neunert C, Noroozi N, Norman G, et al. Severe bleeding events in adults and children with primary immune thrombocytopenia: a systematic review. J Thromb Haemost. 2015;13(3):457-464. doi: $10.1111 /$ jth. 12813 .
35. Arnold DM, Donahoe L, Clarke FJ, et al. Bleeding during critical illness: a prospective cohort study using a new measurement tool. Clin Investig Med Médecine Clin Exp. 2007;30(2): E93-102.

36. Peberdy MA, Callaway CW, Neumar RW, et al. Part 9: postcardiac arrest care: 2010 American Heart Association Guidelines for Cardiopulmonary Resuscitation and Emergency Cardiovascular Care. Circulation. 2010;122(18 suppl 3):S768-S786. doi:10. 1161/CIRCULATIONAHA.110.971002.

37. Valzani Y, Marudi A, Baroni S, et al. What is the role of head computed tomography in post-resuscitation care. Crit Care. 2014; 18(suppl 1):P486. doi:10.1186/cc13676.

38. Inamasu J, Miyatake S, Suzuki M, et al. Early CT signs in out-ofhospital cardiac arrest survivors: temporal profile and prognostic significance. Resuscitation. 2010;81(5):534-538. doi:10.1016/j. resuscitation.2010.01.012.

39. Kim SH, Choi SP, Park KN, Youn CS, Oh SH, Choi SM. Early brain computed tomography findings are associated with outcome in patients treated with therapeutic hypothermia after out-ofhospital cardiac arrest. Scand J Trauma Resusc Emerg Med. 2013;21:57. doi:10.1186/1757-7241-21-57.

40. Friberg H, Cronberg T, Dünser MW, Duranteau J, Horn J, Oddo M. Survey on current practices for neurological prognostication after cardiac arrest. Resuscitation. 2015;90:158-162. doi:10.1016/ j.resuscitation.2015.01.018.

41. Arnaout M, Mongardon N, Deye N, et al. Out-of-hospital cardiac arrest from brain cause: epidemiology, clinical features, and outcome in a multicenter cohort. Crit Care Med. 2015;43(2): 453-460. doi:10.1097/CCM.0000000000000722.

42. Brouwers PJ, Wijdicks EF, Hasan D, et al. Serial electrocardiographic recording in aneurysmal subarachnoid hemorrhage. Stroke. 1989;20(9):1162-1167.

43. Waydhas C. Intrahospital transport of critically ill patients. Crit Care. 1999;3(5):R83-R89. doi:10.1186/cc362. 